PlastOx 2007 (2009) 191-203

(C) EDP Sciences, 2009

DOI: $10.1051 /$ ptox/2009014

\title{
Électrochimie et corrosion à l'échelle des microstructures
}

\author{
R. Oltra et B. Vuillemin \\ Université de Bourgogne, Institut CARNOT de Bourgogne, UMR 5209 CNRS, Interfaces \\ et Réactivité dans les Matériaux, BP. 47 870, 21078 Dijon Cedex, France \\ e-mail : roland.oltra@u-bourgogne.fr ; bruno.vuillemin@u-bourgogne.fr
}

\begin{abstract}
Résumé. Dans ce chapitre, on montre que le rôle de la microstructure sur les processus de corrosion localisée, par exemple la sensibilité d'un précipité par rapport au reste de la matrice, peut être évalué et simulé en tenant compte des processus de couplage galvanique et de transport des espèces chimiques, grâce à l'évolution récente d'un ensemble de méthodes d'analyses chimiques, électrochimiques et électriques dont la résolution spatiale est adaptée à l'échelle micronique qui caractérise la microstructure des alliages métalliques. Il est possible de disposer, à cette échelle, d'informations comme la différence de potentiel de surface intrinsèque à la coexistence d'une phase et de sa matrice, les cinétiques électrochimiques élémentaires des mêmes phase et matrice, la distribution du courant en solution qui permet de définir la polarisation de surface, l'évolution chimique locale contrôlée par le transport de matière en phase liquide en fonction des réactions de réduction ou de dissolution. Ces informations constituent les données d'entrée des modèles de comportement en corrosion localisée des alliages concernés.
\end{abstract}

\section{POSITION DU PROBLÈME}

Tous les processus de corrosion localisée sont contrôlés par un couplage galvanique qui va intervenir à différentes échelles selon que l'on se préoccupe de la résistance d'un assemblage (millimétrique), de la protection exercée par un revêtement $(100 \mu \mathrm{m})$ ou du rôle des phases précipitées (inclusions, précipités durcissants) qui dans ce dernier cas sera directement relié à la microstructure qui imposera le facteur d'échelle ( $10 \mu \mathrm{m}$ et en dessous). Néanmoins quelle que soit l'échelle critique, du point de vue électrochimique on pourra décrire les phénomènes mis en jeu en se basant sur une approche commune qui repose en fait sur l'analyse d'un problème de corrosion bimétallique. Dans la littérature on trouve donc différents travaux qui proposent une analyse mathématique très générale des ces processus de corrosion bimétallique, depuis les travaux les plus anciens, ceux de Wagner [1] et Waber [2] en particulier, jusqu'à ceux plus récents de Smyrl [3, 4]. Ces travaux sont à l'origine de l'accroissement des travaux de simulation mais également du développement des sondes locales qui permettent de concentrer les analyses à l'échelle critique des effets engendrés par les hétérogénéités microstructurales. Ce que montrent toutes ces approches, c'est quelle que soit l'échelle, et en particulier pour les effets microstructuraux, il suffit de définir comment fonctionne la cellule élémentaire concernée. Comme le montre le schéma (Fig. 1) on voit que la géométrie jouera un rôle majeur, mais en fait ce facteur géométrique conditionne le couplage du contrôle des distributions de courant (électrostatique) avec le contrôle du transport des espèces chimiques mises en jeu dans la cellule électrochimique élémentaire considérée.

Quels sont les paramètres essentiels qui contrôlent donc le comportement de la cellule de couplage bimétallique (que l'on soit à l'échelle de la microstructure ou non) :

- géométrie :

- taille des composantes élémentaires du couple: à la Fig. 1c on voit l'importance de ce facteur si l'on revient au sujet de cet exposé (à l'échelle de la microstructure on devra s'intéresser à la sensibilisation de l'interface matrice - précipité), 


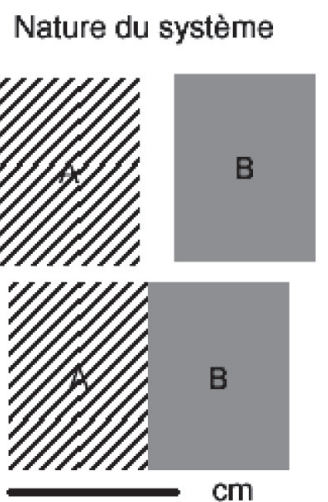

(a)

\section{Endommagement}

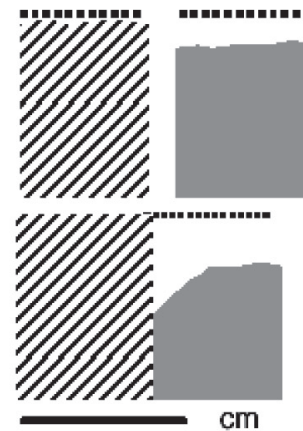

(b)
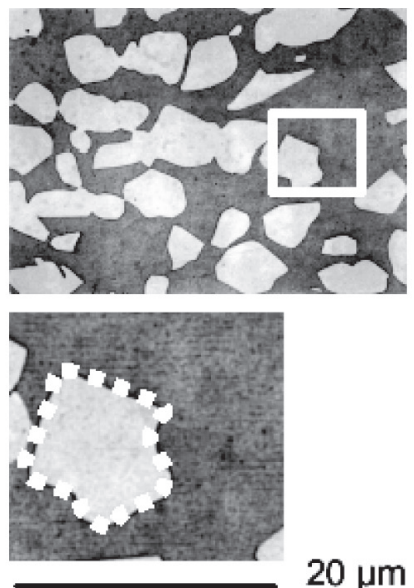

$20 \mu \mathrm{m}$

(c)

Figure 1. Mise en évidence du rôle du facteur d'échelle sur les processus de corrosion bi-métallique. On constate que l'évaluation du seul couplage (a) ne permet pas d'évaluer l'endommagement réel (b). Par conséquent sur une microstructure réelle la corrosion se localisera à la frontière entre phase (claire) et matrice (foncée), en supposant une phase précipitée aynat un comportement cathodique par rapport à la matrice.

- épaisseur de l'électrolyte : paramètre très important si l'on décrit les phénomènes de corrosion en milieu confiné (crevasse, corrosion intergranulaire, corrosion fissurante, corrosion atmosphérique, ...).

- les propriétés de l'électrolyte :

- en tout premier, sa conductivité car elle conditionne la distribution du courant de couplage en solution (ceci est décrit dans l'annexe I),

- sa composition et les réactions associées au transport des espèces mises en jeu lors de la réaction de corrosion,

- les cinétiques électrochimiques associées à chacun des membres du couple.

On a l'habitude d'évaluer la corrosion bimétallique par l'amplitude de la force motrice du couplage associée aux différences des potentiels électrochimiques des deux demi-piles qui constituent l'assemblage (approche thermodynamique - échelles des couples galvaniques) mais en réalité l'endommagement réel sera contrôlé par la combinaison simultanée des transferts de charge et de matière dans la cellule élémentaire électrochimique représentative. Si on se place à l'échelle de la microstructure, on peut schématiquement, comme le montre la Fig. 2, représenter l'ensemble des phénomènes qui contrôlent tout phénomène de corrosion localisée associée à la microstructure. Sur cette figure, on représente l'évolution d'une corrosion locale qui aurait pris naissance à cause de la sensibilité d'un précipité par rapport au reste de la matrice. De façon très schématique, on considère que la dissolution anodique de la phase en question est couplée à une réduction par exemple de l'oxygène présent en solution sur la matrice avoisinante. On peut ainsi mettre en lumière :

- le contrôle de la distribution du courant dans l'électrolyte (voir à l'annexe I la définition de la portée du couplage) : on comprend que les lignes de courant doivent boucler en obéissant aux lois de l'électrostatique (l'électrolyte est un conducteur ionique),

- le gradient de potentiel en surface fixe la vitesse des réactions qui s’y déroulent $[5,6]$,

- les gradients de concentration des espèces, contrôlés par le transport de matière (diffusion, migration, voire convection) qui peuvent affecter les cinétiques de corrosion à cause de leur localisation : c'est particulièrement le cas pour la réaction cathodique qui va conduire à une alcalinisation de 


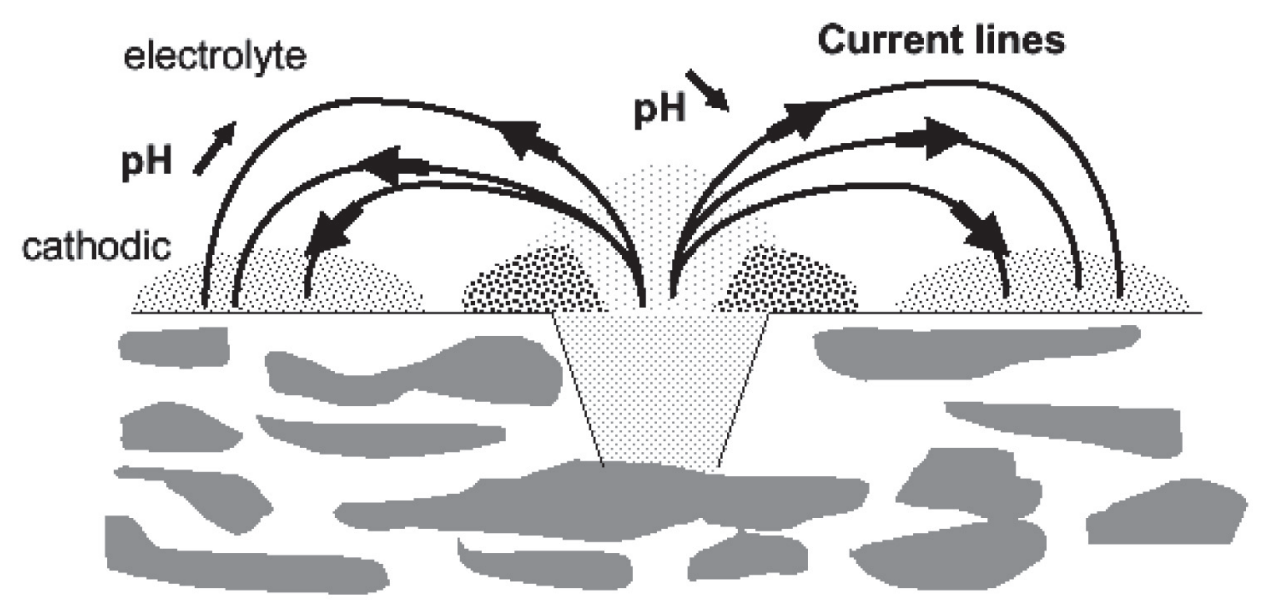

Figure 2. Description schématique des processus contrôlant le couplage à l'échelle de la microstructure.

la surface de l'électrode concernée par la réaction cathodique (le $\mathrm{pH}$ augmente). Il en est de même pour l'acidification liée à l'hydrolyse des cations métalliques à l'aplomb de la zone de dissolution.

\section{APPROCHES EXPÉRIMENTALES À L'ÉCHELLE DE LA MICROSTRUCTURE}

D'un point de vue expérimental, il est donc nécessaire de faire évoluer les méthodes d'analyses chimiques et électrochimiques pour disposer des données d'entrée des modèles prédictifs (voir paragraphe 3) d'une part et de méthodes de validation des simulations d'autre part. Un grand nombre de données ou paramètres sont effectivement nécessaires à évaluer, mais on peut en sélectionner quelques unes qui sont indispensables pour déterminer les risques de corrosion associés à une microstructure donnée (on considère une cellule élémentaire constituée d'une phase et d'une matrice) :

- la force électromotrice intrinsèque à la coexistence d'une phase et de la matrice environnante,

- les cinétiques électrochimiques élémentaires de la phase et de la matrice,

- la distribution du courant en solution qui permet de définir la polarisation de surface (et donc la densité de courant à la surface),

- l'évolution chimique localisée contrôlée par le transport de matière en phase liquide en fonction des réactions de réduction ou de dissolution.

Dans la suite, on montrera qu'il existe des méthodes bien adaptées pour répondre à ces questions mais elles n'ont pas toutes été validées sur des microstructures réelles (on fixe comme ordre de grandeur caractéristique l'échelle de $10 \mu \mathrm{m})$.

\subsection{Comment évaluer la force électromotrice intrinsèque à la coexistence d'une phase et de sa matrice : méthode SKPFM (Scanning Kelvin Probe Force Microscopy)}

Parmi les techniques de caractérisation électrique en champ proche, la microscopie à force atomique en mode Kelvin (KFM) est celle dont l'intérêt va croissant au cours des années (elle a été mise au point en 1991 et aujourd'hui des équipements commerciaux permettent de réaliser des mesures à l'échelle submicronique). Cette technique dérive directement de la sonde de Kelvin (KP) qui permet la mesure du potentiel de sortie. Le potentiel de sortie dépend de phénomènes physico-chimiques de surface (activité catalytique, adsorption, reconstruction de surface, piégeage de charge en surface, comportement semiconducteur d'une surface, ... ). 


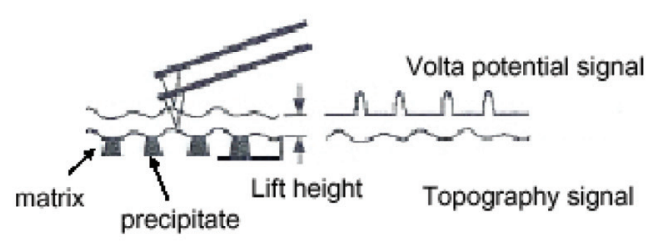

(a)

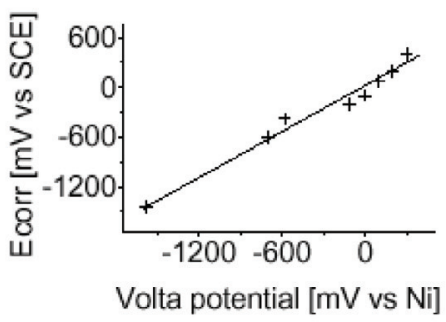

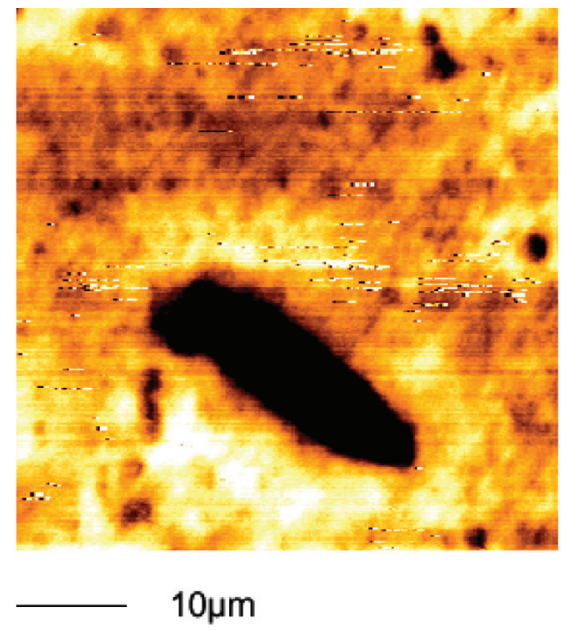

$\Delta$ (précipité - matrice) $\sim 470 \mathrm{mV}$

(b)

Figure 3. (a) : Principe de fonctionnement de la sonde SKPFM - (b) : relation de proportionnalité entre le potentiel de corrosion libre (mV/SCE) et le potentiel de Volta ( $\mathrm{mV}$ vs $\mathrm{Ni}$ ) - (c) : exemple de mesure SKPFM sur un IM (alliage 2xxx) montrant qu'il est plus noble que la matrice (différence de potentiel de Volta de $470 \mathrm{mV}$ ).

La méthode SKPFM est donc une technique AFM utilisant une pointe conductrice où la différence de potentiel entre cette pointe et la surface peut être mesurée de la même façon qu'avec une sonde de Kelvin classique. Par rapport à une mesure topographique AFM, le levier n'est pas piloté par le piezo-électrique à sa fréquence de résonance mais par une tension sinusoïdale (AC) appliquée à cette même fréquence de résonance. Dans cette configuration, la topographie et le potentiel de surface sont déterminés simultanément. C'est donc une caractéristique électrique complexe de la surface mais qui a néanmoins été identifiée comme pouvant être associée au comportement en corrosion (en particulier au potentiel de corrosion) des matériaux métalliques [7]. En résumé, comme le montre la Fig. 3, on peut établir une relation entre la force motrice du couplage en milieu corrosif (différence entre les potentiels de corrosion des phases) et la différence de potentiel de surface mesuré par la sonde SKPFM ou KFM, en prenant comme référence, le potentiel e surface d'un métal pur (dans l'exemple cité, le Nickel).

\subsection{Comment mesurer les cinétiques électrochimiques élémentaires de la phase et de la matrice : la microélectrochimie}

La méthode dite "Microcell technique" développée par Suter et Böhni [8] se compose en fait d'une cellule électrochimique classique à trois électrodes, la seule différence étant la taille de la cellule. Celleci se termine par un capillaire étiré de faible diamètre $(10 \mu \mathrm{m}$ à $100 \mu \mathrm{m})$ qui est plaqué sur la zone de l'électrode à étudier, l'étanchéité du contact étant assurée par un polymère déposé à l'extrémité du capillaire. Comme le montre la figure 4a, le capillaire est solidaire d'une tourelle de microscope et l'on peut ainsi repérer préalablement par un examen microscopique classique la zone à caractériser (Fig. 4b). Cette technique nécessite un système de polarisation (potentiostat) qui permette d'imposer et de détecter des courants très faibles (de l'ordre du nA).

Cette méthode a été appliquée, par exemple, à l'étude de la sensibilité à la piqûre d'inclusions $\mathrm{MnS}$ dans les aciers inoxydables. Un très important travail de pionnier dans ce domaine est à mettre à l'actif de T. Suter sur l'origine des mécanismes locaux mis en jeu lors de l'amorçage des piqûres pour des aciers inoxydables austénitiques [9]. Cette approche locale individuelle peut s'appliquer tant 


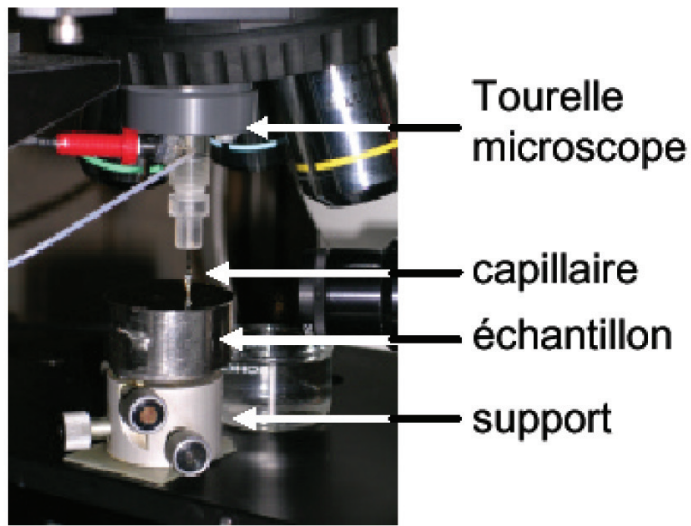

(4a) Vue du dispositif

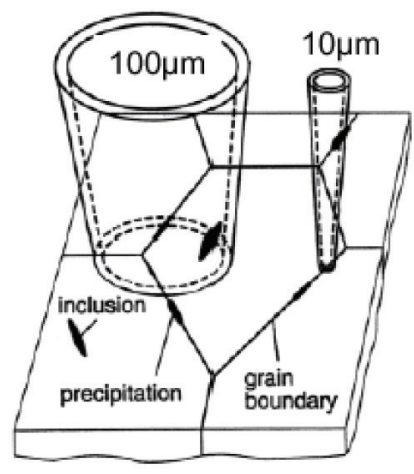

(4b) Principe

Figure 4. Dispositif et illustration des capacités d'analyse par microélectrochimie à cellule capillaire.

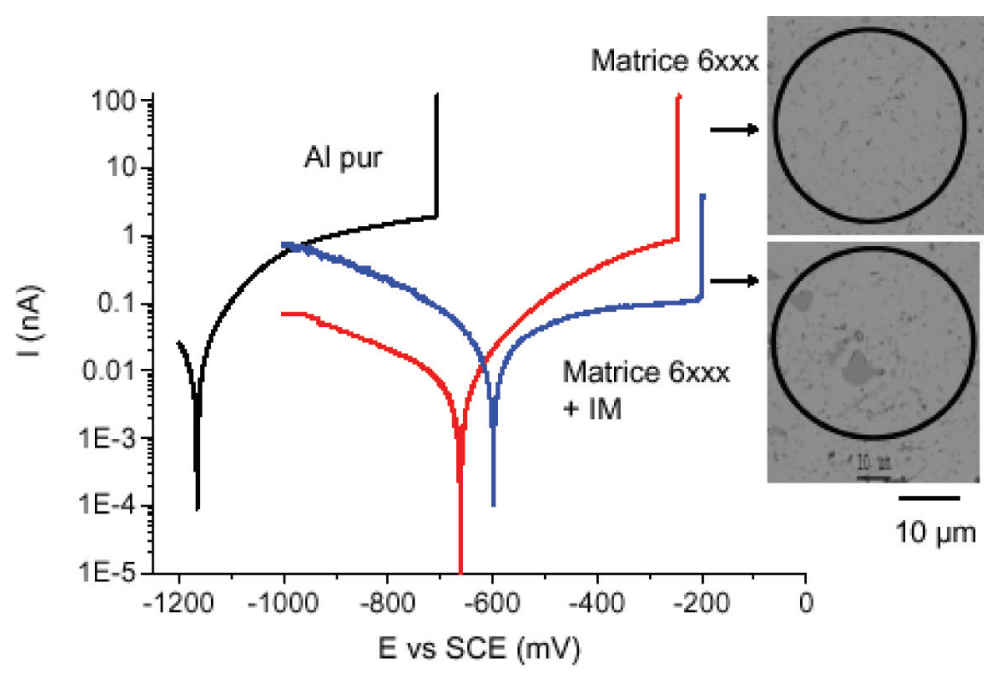

Figure 5. Exemple de mesure électrochimique locale - capillaire $50 \mu \mathrm{m}$ - mettant en évidence le comportement "cathodique" d'un intermétallique ( $\mathrm{Al}, \mathrm{Fe})$ dans un alliage d'aluminium 6xxx et montrant la réactivité de la matrice.

sur des microstructures réelles comme le montre la figure 5, pour un alliage d'aluminium 6xxx, que sur des microstructures modèles N. Birbilis et coll. [10]. Ces auteurs ont pu définir une base de données exhaustive concernant le comportement électrochimique des intermétalliques contenus dans les alliages de type 7xxx et ainsi distinguer les intermétalliques nobles avec une haute activité électrochimique, par exemple $\mathrm{Al}_{2} \mathrm{Cu}, \mathrm{Al}_{7} \mathrm{Cu}_{2} \mathrm{Fe}$, qui sont susceptibles de causer des piqûres et les intermétalliques nobles avec une faible activité électrochimique comme $\mathrm{Al}_{3} \mathrm{Zr}$, peu susceptibles de causer des piqûres.

\subsection{Comment imager la distribution du courant en solution : la technique SVET (Scanning Vibrating Electrode Technique)}

La mesure des courants locaux peut être effectuée avec un système SVET (le dispositif le plus performant est commercialisé par Applicable Electronics). Cette technique mise au point par Jaffe et Nuccitelli [11] pour des études biocellulaires a été reprise et adaptée par Isaacs [12] pour l'étude de la 
corrosion localisée et permet dans ce cas de mesurer les densités de courant au dessus d'une surface métallique en solution. La sonde de mesure est constituée d'une microélectrode en Platine Iridium recouverte à son extrémité d'un dépôt de noir de platine. Le diamètre de la sphère de ce dépôt est d'environ 15 à $20 \mu \mathrm{m}$ ce qui correspond à une capacité de $10 \mathrm{nF}$. Deux piézo-électriques imposent à la microélectrode des vibrations d'environ $20 \mu \mathrm{m}$ d'amplitude et de fréquence respectivement de $600 \mathrm{~Hz}$ et $200 \mathrm{~Hz}$ dans les directions parallèle et normale à la surface de l'échantillon. La chute de potentiel qui est mesurée par la microélectrode est amplifiée et convertie en densité de courant (la loi d'Ohm), après une calibration dans une solution de conductivité connue. Le déplacement de la microélectrode est effectué par un micromanipulateur motorisé, contrôlé par ordinateur et dont le pas minium réalisable est de $0.5 \mu \mathrm{m}$.

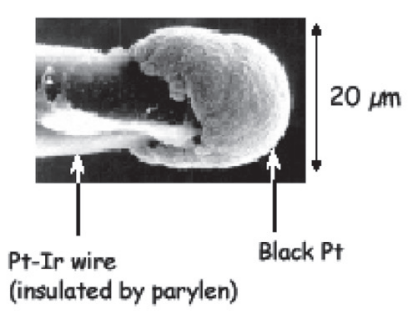

(a)

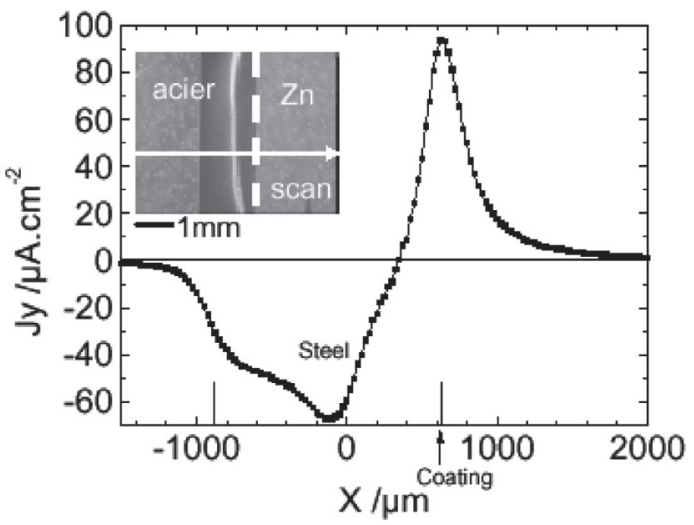

(b)

Figure 6. (a) : Vue de la partie active de la sonde - (b) : distribution des courants mesurée à $150 \mu \mathrm{m}$ de la surface de la tranche d'un acier galvanisé.

L'exemple choisi pour illustrer le potentiel de cette technique n'est pas directement lié à l'analyse des effets de couplage associé à une microstructure particulière mais à celui existant sur la tranche d'un matériau revêtu, en l'occurrence un acier galvanisé. Si l'on effectue des balayages au dessus de la surface, qui comporte donc une zone "anodique" (revêtement de $\mathrm{Zn}$ de quelques micromètres d'épaisseur) et une zone "cathodique" (l'acier), on peut déterminer la distribution du courant dans l'électrolyte. Pour évaluer la densité de courant à la surface il convient de modéliser la réponse et de calculer la densité de courant réelle qui ne peut être mesurée directement [13].

\subsection{Comment suivre l'évolution chimique par des sondes locales (méthodes de chimie ou d'électrochimie analytiques)}

En corrosion, ce sont les gradients de pH qui sont le plus étudiés: en effet comme le montre la figure 2, l'acidification et/ou l'alcalinisation locale renseigne directement sur la localisation des réactions et les changements de phase possibles soit en surface (précipitation) ou en volume (dégagement gazeux) qui pourront par exemple être définis par des approches à l'état stationnaire assez proches de celle utilisée pour définir les diagrammes de Pourbaix (voir paragraphe modélisation).

\subsubsection{Mesures ampérométriques : Le microscope électrochimique (SECM-Scanning Electrochemical Microscope)}

Cette technique, développée par Bard [14] est une technique d'analyse électrochimique basée sur l'utilisation d'une ultramicroélectrode qui va constituer une sonde locale pour mesurer, dans sa version 


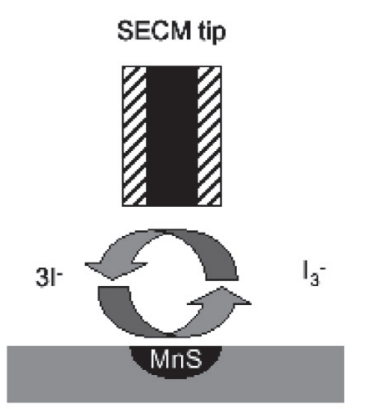

(a)

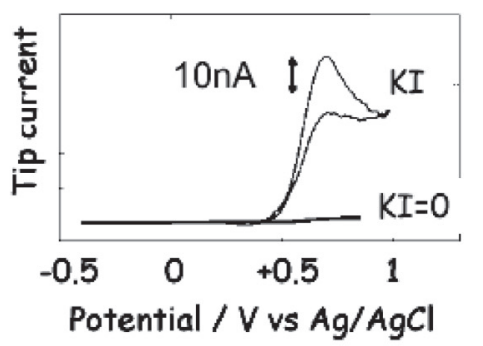

(b)

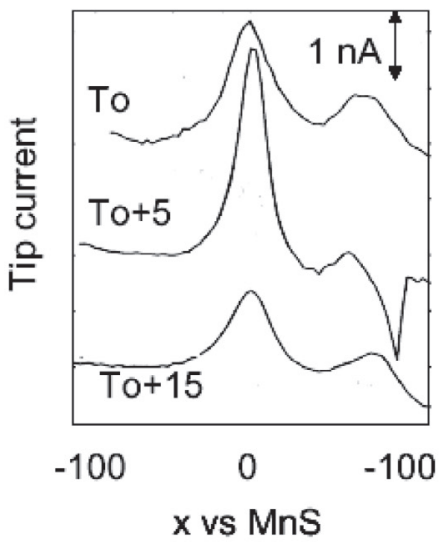

(c)

Figure 7. (a) : Schéma de fonctionnement de la sonde SECM - (b) : étalonnage de la pointe SECM - (c) : mesure du courant en mode positive feedback à l'aplomb de l'inclusion $\mathrm{MnS}$ qui se dissout en quelques minutes.

la plus classique, le courant continu généré par l'électrolyse d'une espèce électroactive en solution. La technique SECM est utilisée selon deux modes :

- dans le premier elle requiert l'utilisation d'un médiateur chimique, espèce électroactive ajoutée dans la solution. On utilise un bipotentiostat qui permet de contrôler indépendamment le potentiel de l'électrode sonde et celui de la surface étudiée. Quand la sonde s'approche d'une surface conductrice le courant mesuré par la sonde augmente à cause de la régénération de l'espèce électroactive (positive feedback). Si le substrat est isolant, le courant dans la sonde diminue à cause du blocage de la diffusion de l'espèce électroactive (negative feedback).

- dans le mode 'génération/collection', l'espèce électroactive n'est pas ajoutée à la solution mais résulte des réactions chimiques ou électrochimiques qui se déroulent à la surface du substrat. Les variations du courant «collecté » au niveau de l'électrode dépendent de la nature chimique des espèces produites et de la vitesse à laquelle elles sont produites. Ce mode permet, par exemple, d'obtenir des informations sur la cinétique des réactions d'oxydoréduction lors de la phase de croissance des piqûres et d'identifier les produits de corrosion [15].

Dans les deux cas, la calibration de la sensibilité de la microélectrode qui sert d'électrode analytique repose sur le contrôle diffusionnel lié au rapport de la distance sonde /surface et à la taille de l'ensemble microélectrode (c'est-à-dire la taille de la microélectrode mais également celle de son enrobage) comme le montre la Figure 7(a). Par contre on n'a peu d'exemple de son utilisation en relation avec le rôle de la microstructure sur la corrosion localisée. On peut citer les travaux de R.C. Alkire et coll. [16] qui ont permis de tester les différentes hypothèses relatives au mode de dissolution des inclusion de $\mathrm{MnS}$ (ces inclusions contenant par ailleurs des éléments métalliques) afin de définir quelle espèce chimique soufrée était responsable de la corrosion par piqûre des aciers inoxydables associée à la dissolution des inclusions de MnS. Dans ce cas l'électrode-sonde du SECM était une fibre de carbone et le médiateur le couple (iodure/iodate) $\mathrm{I}^{3-} / \mathrm{I}^{-}$. Comme en solution, les espèces soufrées venant de la dissolution des inclusions $\mathrm{MnS}$ pouvaient réagir avec le couple $\mathrm{I}^{3-} / \mathrm{I}$ selon les réactions suivantes :

$$
\begin{gathered}
\mathrm{I}^{3-}+2 \mathrm{~S}_{2} \mathrm{O}_{3}^{2-} \rightarrow 3 \mathrm{I}^{-}+\mathrm{S}_{4} \mathrm{O}_{6}^{2-} \\
\text { ou } \mathrm{I}^{3-}+\mathrm{HS}^{-} \rightarrow 3 \mathrm{I}^{-}+\mathrm{H}^{+}+\mathrm{S}
\end{gathered}
$$



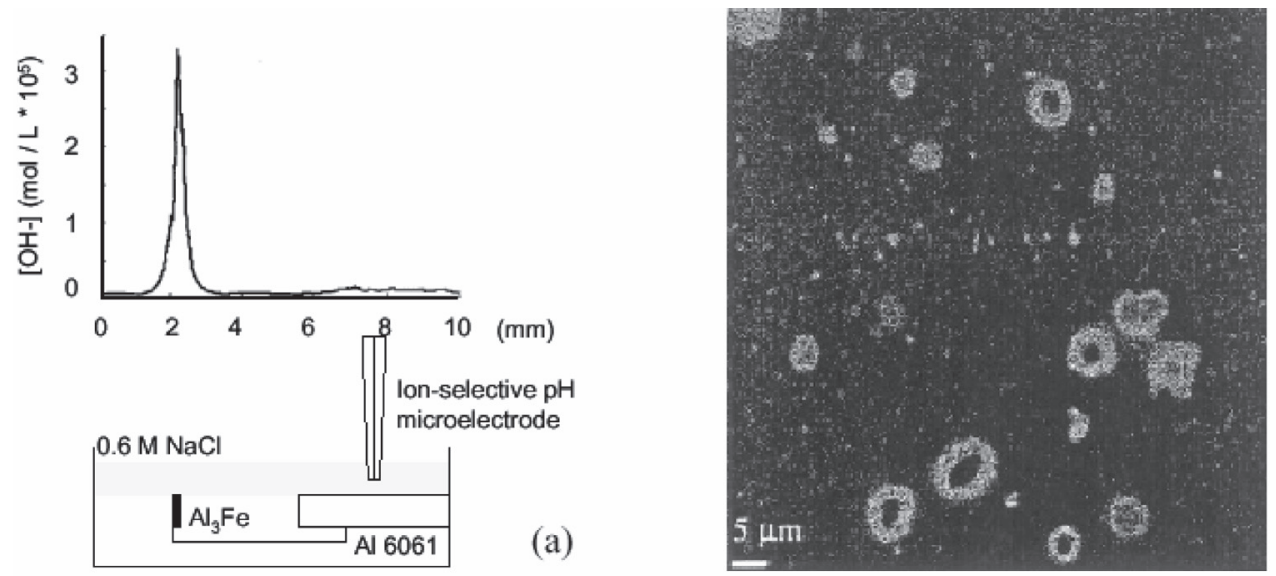

(b)

Figure 8. (a) : Développement d'une sonde de pH pour évaluer le changement de pH sur une électrode modèle [18] (b) : mesure par fluorescence en microscopie confocale sur un alliage d'aluminium [19].

le suivi de la réaction à l'électrode sonde SECM (Fig. 7(a)), en mesurant le courant en mode «positivefeedback » :

$$
3 \mathrm{I}^{-} \rightarrow \mathrm{I}^{3-}+2 \mathrm{e}^{-}
$$

a permis de confirmer la formation de thiosulfates, lors de la dissolution de $\mathrm{MnS}$, selon la réaction:

$$
2 \mathrm{MnS}+3 \mathrm{H}_{2} \mathrm{O} \rightarrow 2 \mathrm{Mn}^{2+}+\mathrm{S}_{2} \mathrm{O}_{3}^{2-}+6 \mathrm{H}^{+}+8 \mathrm{e}^{-}
$$

l'analyse SECM a permis de valider l'hypothèse de l'empoisonnement de la surface par le soufre, celuici pouvant en effet se former par dismutation des ions thiosulfates selon :

$$
\mathrm{S}_{2} \mathrm{O}_{3}^{2-}+\mathrm{H}_{2} \mathrm{O} \rightarrow \mathrm{S}_{\text {ads }}+\mathrm{SO}_{4}^{2-}+2 \mathrm{H}^{+}+2 \mathrm{e}^{-} .
$$

\subsubsection{Mesures potentiométriques : sondes de $\mathrm{pH}$}

Les sondes locales de $\mathrm{pH}$ peuvent être, par exemple, constituées d'un microcapillaire étiré dont l'extrémité est remplie d'un composé liquide ionophore $\left(\mathrm{H}^{+}\right.$pour le $\left.\mathrm{pH}\right)$ formant une membrane sélective d'ions. Celle-ci est en contact d'un côté avec l'électrolyte à analyser et de l'autre côté avec une solution aqueuse de référence (de concentration connue et tamponnée). La différence de potentiel à travers la membrane est liée à l'activité dans l'électrolyte de l'ion qu'elle transporte. Elle est mesurée par rapport à une électrode de référence (par exemple $\mathrm{Ag} / \mathrm{AgCl}$ ) introduite dans le capillaire.

A titre d'exemple, on peut citer le dispositif proposé par l'équipe de R.C. Alkire [18] qui permet d'avoir une résolution horizontale de $2 \mu \mathrm{m}$ et verticale de $1 \mu \mathrm{m}$. Les auteurs ont mesuré l'évolution du $\mathrm{pH}$ au-dessus d'une cellule galvanique composée d'une électrode d' $\mathrm{Al}_{3} \mathrm{Fe}$ et d'un échantillon d' $\mathrm{Al}$ 6061. Ils ont pu observer une augmentation du $\mathrm{pH}$ au-dessus de l'électrode d' $\mathrm{Al}_{3} \mathrm{Fe}$, qui explique son rôle cathodique et le fait que la réaction de réduction de l'oxygène produise des espèces $\mathrm{OH}^{-}$ autour de l'inclusion dans le cas d'une solution aérée (Fig. 8a). Ils en déduisent les différentes étapes du mécanisme de corrosion de l'alliage $\mathrm{Al} 6061$ autour des intermétalliques d' $\mathrm{Al}_{3} \mathrm{Fe}$ dans un milieu chloruré. 


\subsubsection{Mesures optiques}

Si l'on s'intéresse au rôle de la microstructure, on peut citer quelques exemples d'application des méthodes optiques:

a) Microscopie Laser confocale (CLSM) : largement utilisée en biologie, en mode fluorescent elle permet d'évaluer et non de quantifier réellement des changements de pH. R.W. Smyrl [19] a étudié par CLSM la surface des échantillons d'alliages d'aluminium 2024 et 6061 après corrosion (mode ex-situ). Dans le cas du 6061 la fluorescence est émise de façon uniforme au dessus des inclusions actives composées d'Al-Mg-Si (Fig. 8b). La fluorescéine est emprisonnée dans les produits de corrosion qui forment un relief et sont visibles. C'est l'élément $\mathrm{Mg}$ qui semble se dissoudre préférentiellement dans les conditions d'exposition.

b) Fibre optique fonctionnalisée (optode) : S. Szunerits et D. Walt [20] ont utilisé plutôt qu'une seule fibre un faisceau de fibres fonctionnalisées (diamètre unitaire des fibres de quelques $\mu \mathrm{m}$ donnant un faisceau de fibre de $350 \mu \mathrm{m}$ ). A l'extrémité des fibres on dépose un polymère dans lequel est piègé la molécule fluorescente En utilisant un autre composé fluorescent greffé à la fibre, la morine, ils ont pu imager la concentration des ions $\mathrm{Al}^{3+}$ et cela in-situ.

c) Electrolyte gélifié : il est intéressant de mentionner qu'au delà de l'échelle de la microstructure, par exemple pour évaluer la corrosion sur les différentes zones concernées par une soudure, certains auteurs ont montré qu'il était possible d'effectuer des essais de corrosion non en solution mais en présence d'un gel contenant un indicateur coloré [21]. Ceci permet de révéler les changements de $\mathrm{pH}$ associé aux réactions anodiques et cathodiques semblables à ceux décrits à la Figure 2.

\section{MODÉLISATION DU ROLE DE LA MICROSTRUCTURE EN CORROSION LOCALISÉE}

La modélisation des phénomènes de corrosion localisée associés à la microstructure consiste à mettre en équation le comportement de la cellule élémentaire telle qu'elle est décrite à la Fig. 1(c). Ceci a été clairement démontré par les travaux de Smyrl et Morris [3] mais dans leur démonstration les auteurs n'ont tenu compte que de l'aspect couplage (distribution des lignes de courant) et en réalité il faut considérer également les évolutions chimiques associées aux réactions mises en jeu. On doit par exemple, tenir compte des gradients de concentration en oxygène sur la cinétique de la réaction cathodique afin de décrire l'influence du transport de masse sur la réaction cathodique. Dans le cas où la réduction de l'oxygène n'est pas limitée par le transfert de charge mais par la diffusion dans l'électrolyte, ce qui impose un contrôle cathodique du couplage galvanique entre les éléments du couple concerné (matrice / phase précipitée), on comprend mieux l'intérêt de disposer de méthodes de simulation faisant appel au couplage multi-physique. La modélisation permet soit de simuler correctement les mesures expérimentales obtenues, par exemple avec la technique SVET, mais également de prévoir le comportement électrochimique dans des conditions d'emploi difficiles à analyser : par exemple pour des couches minces d'électrolyte se rapprochant d'une situation de corrosion atmosphérique.

La définition des modèles de corrosion reste très générale et le fait que l'on étudie le rôle de la microstructure ne fait que fixer les échelles spatiales des phénomènes. Ceci conduit donc à formaliser une description de base du maillage de la cellule type dont on modifiera les proportions en fonction des cas à traiter (Fig. 9) si on réalise une simulation par la méthode des éléments finis (FEM). Dans la modélisation, la première étape consiste donc à représenter la cellule élémentaire (cellule par exemple décrite à la Figure 2) de façon à pouvoir résoudre numériquement le système d'équations qui va déterminer son comportement. En fait quel que soit le cas de corrosion à simuler, le comportement de chacune des espèces en solution dans le volume d'électrolyte associé à la cellule de corrosion, sera régi par la loi de Nernst-Planck, qui traduit l'évolution des concentrations suivant 3 processus: la diffusion, l'électromigration et la production/consommation d'espèces au sein du volume, les phénomènes de convection étant négligés. La simulation, soit à l'état stationnaire soit en dynamique, 


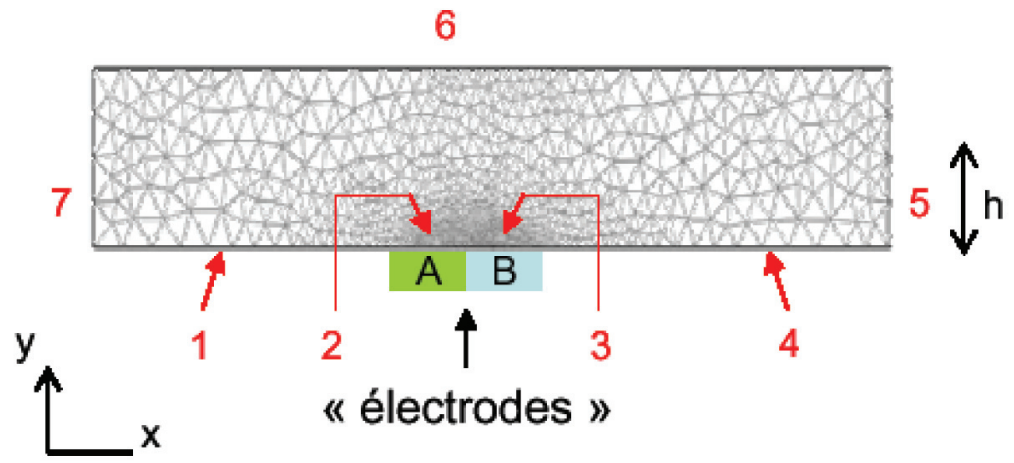

Figure 9. Exemple de description d'une géométrie utilisée pour la simulation en deux dimensions d'un processus de corrosion galvanique (électrodes A et B).

s'apparente mathématiquement à la résolution du système d'équations ci-dessous, expressions traduisant le comportement de chaque espèce $i(i=1, \ldots, n)$ en solution :

terme de diffusion

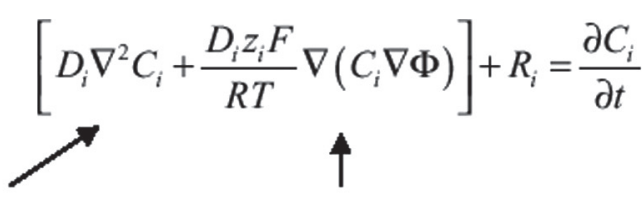

terme d'électromigration

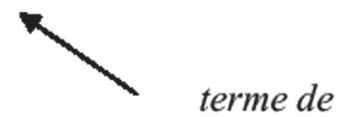

production/consommation

Enfin, le potentiel électrostatique $\Phi$ de la solution est déterminé moyennant l'hypothèse que l'électroneutralité de la solution est vérifiée en tout point :

$$
\sum_{i} z_{i} C_{i}=0
$$

L'une des étapes-clé consiste à exprimer le terme $\mathrm{R}_{\mathrm{i}}$ de production/consommation de l'espèce i qui dépend non seulement des réactions chimiques homogènes prises en compte dans la solution, mais aussi des réactions électrochimiques localisées à l'interface métal/solution [22].

Il faut définir les conditions aux frontières qui s'écrivent d'une façon très générale :

- Frontières 1, 4, 5, 7 : conditions d'isolation pour le courant électrique et les flux d'oxygène

- Frontières 2 et 3 : ce que l'on connaît le mieux, les relations courant $=\mathrm{f}$ (potentiel), qui vont permettre d'exprimer le flux de matière produit et / ou consommé (loi de Butler-Volmer).

- Frontière 6 : à l'interface air/solution on a une condition d'isolation pour le potentiel électrique et flux d'oxygène lié à sa dissolution dans la solution. La hauteur $h$, l'épaisseur d'électrolyte considérée, est susceptible de varier. Le maillage est affiné au niveau des électrodes de manière à ce qu'il y ait le maximum d'éléments de maillage à la frontière.

A titre d'exemple, la figure 10 illustre en quoi la simulation peut être utile pour confirmer des mécanismes associés à la microstructure. Dans ce cas on cherche à confirmer le changement de pH associé à la localisation de la réaction cathodique sur un intermétallique présent dans un alliage d'aluminium (pour l'exemple alliage 6xxx et intermétallique de type Al-Mn-Fe-Si-Cr). Dans la définition de l'électrode modèle, le système se limite à un intermétallique au contact d'une portion de matrice. Les caractéristiques électrochimiques des deux phases sont extraites des courbes de polarisation obtenues par microcellule électrochimique (Figure 5). La résolution de l'équation de Nernst-Planck dans l'électrolyte avec les conditions aux limites décrites à la figure 9 , montre un changement de $\mathrm{pH}$ sur une 


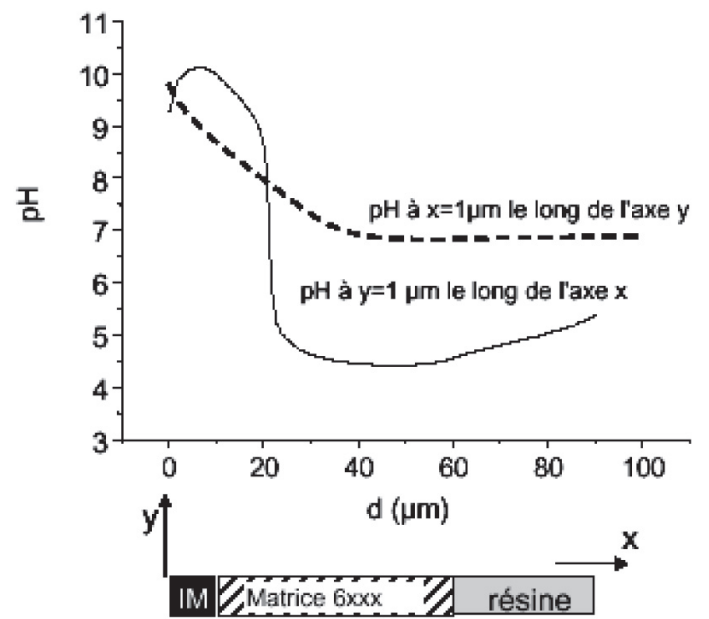

Figure 10. Simulation de la variation de $\mathrm{pH}$ (sur une électrode modèle représentant un intermétallique (siège de la réaction cathodique) et la matrice (alliage 6xxx). La courbe en trait plein représente le profil de $\mathrm{pH}$ au-dessus de l'électrode $(\mathrm{à} \mathrm{y}=1 \mu \mathrm{m})$ tandis que la courbe en trait pointillé montre la décroissance du $\mathrm{pH}$ à l'aplomb de la particule intermétallique quand on s'éloigne de la surface de l'électrode (le $\mathrm{pH}$ de la solution est de 7).

zone qui va au-delà de la particule à cause des processus de transport. La simulation peut donc permettre de représenter ce phénomène pour diverses conditions d'essai (hauteur d'électrolyte, conductivité,....).

\section{CONCLUSION}

Dans ce chapitre, on montre que le rôle de la microstructure sur les processus de corrosion localisée, par exemple la sensibilité d'un précipité par rapport au reste de la matrice, peut être évalué et simulé en tenant compte des processus de couplage galvanique et de transport des espèces chimiques, grâce à l'évolution récente d'un ensemble de méthodes d'analyses chimiques, électrochimiques et électriques dont la résolution spatiale est adaptée à l'échelle micronique qui caractérise la microstructure des alliages métalliques. Il est possible de disposer d'informations à cette échelle comme :

- la force électromotrice intrinsèque à la coexistence d'une phase et de sa matrice estimée par la mesure des potentiels de surface,

- les cinétiques électrochimiques élémentaires de la phase et de la matrice,

- la distribution du courant en solution qui permet de définir la polarisation de surface (et donc la densité de courant à la surface),

- l'évolution chimique localisée contrôlée par le transport de matière en phase liquide en fonction des réactions de réduction ou de dissolution, qui constituent les données d'entrée des modèles de comportement des alliages concernés.

\section{Remerciements}

Les auteurs remercient les centres de recherches des groupes ALCAN, ANDRA et ARCELOR MITTAL pour leur soutien et Florian Thebault, Nicolas Murer et Fabien Rechou pour leur contribution expérimentale.

\section{Références}

[1] Wagner C., J. Electrochem Soc., 98 (1951) 116.

[2] Waber T., J. Electrochem J. Soc., 103 (1956) 567. 
[3] Morris R., Smyrl W., J. Electrochem Soc., 136 (1989) 3237.

[4] Alodan M.A. and Smyrl W.H., J. Electrochem Soc., 144 (1997) L282.

[5] Vetter K.J., dans Electrochemical Kinetics Theoretical and Experimental Aspects, Academic Press, New-York, London, (1967), p.746

[6] Oltra R., Keddam M., dans Les Aciers Inoxydables, Eds. Baroux B., Beranger G., Lacombe P., Editions de Physique, 1990, p.215

[7] Schmutz P., Frankel G.S., J. Electrochem J. Soc., 145 (1998) 2285.

[8] Suter T., Bohni H., Electrochimica Acta 40 (1995) 1361.

[9] Webb E.G., Suter T., Alkire R.C., J. Electrochem Soc. 148 (2001) B186.

[10] Birbilis N, Buchheit R.G., J. Electrochem Soc., 152 (2005) B140.

[11] Jaffe L.F., Nuccitelli R., Journal Cell. Bio., 63 (1974) 614.

[12] Isaacs H.S., Corr. Sci., 28 (1988) 547.

[13] Aldykiewicz A.J., Isaacs H.S., Corr. Sci., 40 (1998) 1627.

[14] Bard A.J., Mirkin M.V., Scanning Electrochemical Microscopy, Marcel Dekker Inc., 2001, p. 1

[15] Wipf D.O., Colloids and Surfaces, A: Physicochemical and Engineering Aspects 93, 251(1994)

[16] Paik C.H., White H.S., Alkire R.C., J. Electrochem Soc. 147 (2000) 4120.

[17] Alkire R.C., Lott S.E., J. Electrochem Soc, 136 (1989) 3256.

[18] Park J.O., Paik C.H., Alkire R.C., J. Electrochem Soc., 143 (1996) L174.

[19] Alodan M.A., Smyrl W.H., J. Electrochem Soc., 145 (1998) 1571.

[20] Szunerits S., Walt D.R., Anal. Chem., 74 (2002) p. 886.

[21] Jariyaboon M., Davenport A.J., Ambat R., Connolly B.J., Williams S.W. and Price D.A., Corr. Sci., 49, 877 (2007)

[22] Vuillemin B., Oltra R., Cottis R. and Crusset D., Electrochimica Acta 52 (2007) 7570.

\section{ANNEXE I - THEORIE DE WAGNER}

Un électrolyte n'est pas aussi bon conducteur qu'un métal et la chute ohmique qui existe en solution peut ne pas être négligeable. Cela se traduit par une distribution du potentiel au sein de la solution.

Lors de la définition du potentiel de couplage, on suppose que l'électrolyte est suffisamment conducteur. Dans ce cas, le potentiel est identique sur toute la surface de l'électrode et égale à $\mathrm{E}_{\text {couplage }}$.

Pour une résistance de l'électrolyte importante, le potentiel d'électrode n'est plus constant sur toute la surface. On peut ainsi définir un potentiel de chute ohmique, $\mathrm{E}_{\mathrm{ohm}}$, égal à la différence de potentiel entre les potentiels moyens des deux électrodes [5]. C'est le potentiel correspondant à la chute ohmique en solution. En corollaire, le courant de couplage diminue avec l'augmentation de la résistance de l'électrolyte.

Un critère existe pour savoir s'il existera une variation importante du potentiel d'électrode à la surface. C'est le paramètre de Wagner $W$ ayant la dimension d'une longueur et défini comme égal à la conductivité de l'électrolyte multiplié par la résistance à la polarisation [1]:

$$
W=\sigma\left|\frac{\partial E}{\partial j}\right|
$$

Si $W$ est très inférieur à la longueur caractéristique de la cellule, en général la longueur de l'électrode, le potentiel d'électrode évolue peu à la surface. Alors il est possible de considérer un potentiel d'électrode constant et donc une densité de courant constante sur toute la surface de l'électrode au sein d'un modèle numérique : on a une distribution primaire de courant.

Si $W$ est très supérieur à la longueur caractéristique de la cellule le potentiel d'électrode évolue de manière importante à la surface. Dans ce cas, il est nécessaire de considérer une relation entre le potentiel d'électrode et la densité de courant à la surface. La distribution du courant est dite secondaire. 
On distingue également une troisième catégorie de distribution de courant en solution, dite tertiaire. Elle tient compte de l'influence des variations de concentration en espèces chimiques à la surface de l'électrode sur la distribution du courant.

Les deux premières distributions de courant utilisent la loi de Laplace pour la détermination du potentiel électrique en solution tandis que la dernière nécessite une expression plus générale, la relation de Nernst-Planck (relation (6)). 
